\title{
Industry 4.0 - Potentials for Predictive Maintenance
}

\author{
Zhe Li and Kesheng Wang \\ Department of Production and Quality Engineering \\ Norwegian University of Science and Technology \\ Trondheim, Norway \\ \{zhe.li \& kesheng.wang\}@ntnu.no
}

\author{
Yafei He \\ College of Engineering \\ Shanghai Polytechnic University \\ Shanghai, P.R. China \\ yfhe@sspu.edu.cn
}

\begin{abstract}
Industry 4,0 represents the coming fourth industrial revolution on the way to combine modern industries with Cyber-Physical Systems, Internet of Things and Internet of Services. In an Industry 4.0 factory, machines are connected as a collaborative community to collect, exchange and analyse data systematically. This paper investigates the potentials and trends of predictive maintenance and maintenance management in industrial big data and Cyber-Physical Systems environment. Furthermore, the development of predictive maintenance, its technical challenges, and the potentials under Industry 4.0 era was researched to discover the linkage between Industry 4.0 and predictive maintenance.
\end{abstract}

Keywords- Industry 4.0; industrial big data; predictive maintenance; maintenance management

\section{INTRODUCTION}

The goal of predictive maintenance is to reduce downtime and cost of maintenance under the premise of zero failure manufacturing through monitoring the working condition of equipment and predicting when equipment failure might occur. Prediction for future potential fault allows maintenance to be planned before the fault happens. Ideally, maintenance schedule can be optimized to minimize the cost of maintenance and achieve zero failure manufacturing. However, it is difficult to realize all the advantages of predictive maintenance without the foundation of correlation techniques like big data and cloud-computing. Many manufacturing systems are not ready to manage big data due to the high demands on data access and data quality and use multiple data sources to extract relevant information [1].

Industry 4.0 refers to a fourth generation of industrial activity as a result of the fourth industrial revolution characterized by smart systems and Internet-based solutions [2]. It combines strengths of optimized industrial manufacturing with internet technologies and changes manufacturing process, maintenance strategies and maintenance management significantly. Therefore, many companies face the challenge to assess the diversity of developments and concepts summarized the term Industry 4.0 and to develop their own strategies [3]. However, since lack of research of the potential use of Industry 4.0 and prospect of predictive maintenance, many companies and organizations are exposed to a dilemma, neither to wait too long with their Industry 4.0 implementation nor to start too early and commit fatal errors [4].
Therefore, this paper aims to provide empirical information on the potentials of Industry 4.0 in the research field of maintenance. It may help academics and practitioners to identify and prioritize their steps towards predictive maintenance and condition-based maintenance management under the environment of Industry 4.0.

The remaining part of the paper is organized as follows. Section 2 briefly presents the classification of maintenance strategy and related challenges. Section 3 discusses the related trends accelerated and encouraged under Industry 4.0 era. Section 4 describes a case study, which illustrates how the predictive maintenance and maintenance management can be applied under Industry 4.0 era. Conclusions are summarized in the last section of this paper.

\section{REVIEW OF MAINTENANCE STRATEGIES}

A maintenance action may include a set of maintenance activities: monitoring, condition analysis, routine maintenance, overhaul, repair and rebuilding. According to the European Standard, maintenance is defined as the combination of all technical, administrative and managerial actions during the life cycle of an item intended to retain it in, or restore it to, a state in which it can perform the required function [5]. Progress in maintenance strategy benefits from a long historical development

As shown in Fig. 1, the classification of maintenance strategies can be divided into three classes as corrective maintenance $(\mathrm{CM})$, preventive maintenance $(\mathrm{PM})$, and predictive maintenance (PdM). CM is similar to repair work, which is performed when the equipment has an obvious failure or a breakdown. That is why $\mathrm{CM}$ is also called as run-to failure maintenance, breakdown maintenance or maintenance-onfailure. PM is carried out at predetermined intervals or according to pre-described criteria with the intention to decrease the probability of failure or degradation of certain functions. PM is scheduled without the occurrence of any monitoring activities. The scheduling may be based on the number of working hours or times. These two strategies are very well known in most industries [6]. 


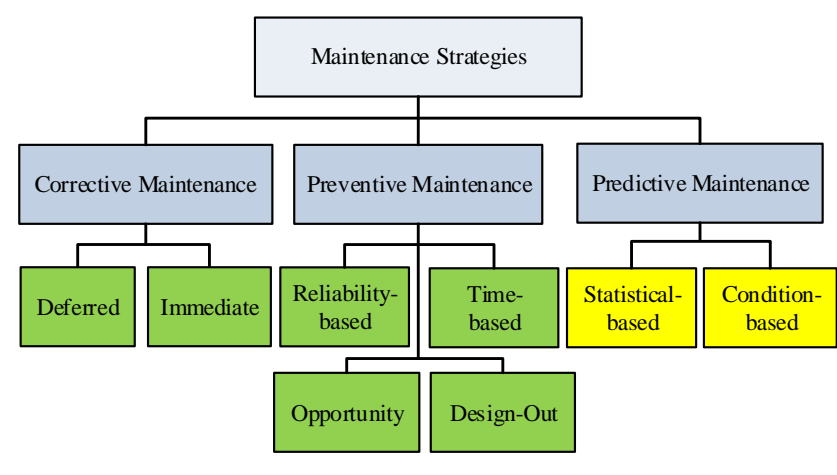

Fig. 1 Classification of maintenance strategies [6].

As one of the most popular and modern maintenance policies, PdM measures parameters in the condition of equipment carry out the appropriate tasks to optimize the service life of machines and processes without increasing the risk of failure [7]. Based on the approaches of measuring the symptom of failures, there are two groups of PdM: statisticalbased PdM and condition-based PdM.

Comparing with other maintenance strategies, PdM has some advantages: equipment that requires maintenance is shut down only before imminent failure; reducing the total time spent maintaining equipment; reducing maintenance costs by avoiding catastrophic damage; increasing availability and reliability of machines; extending life of equipment and processes. However, PdM is facing several challenges as following:

(1) High demands on data access, data quality and data fusion from multiple sources for data sharing and data publication. Since these sources of data often operate in a heterogeneous environment, integration between the systems is problematic [8].

(2) The capability to deal with industrial big data. To leverage big data, industrial businesses need the ability to support different types of information, the infrastructure to store massive data sets, and the flexibility to leverage the information once it is collected and stored - enabling historical analysis of critical trends to enable real-time predictive analysis [9].

(3) The prediction accuracy for PdM. The inaccurate predictive information may result in either unnecessary maintenance, such as early replacement of components, or production downtime because of unexpected machine failures. Therefore, the accuracy of remaining useful life prediction, particularly the long-term prediction, which gives sufficient time to prepare for a maintenance operation, plays an essential role in the full realization of the potentials of predictive maintenance [10].

\section{FROM THE PERSPECTIVE OF MAINTENANCE TOWARDS INDUSTRY 4.0}

Industry 4.0 is the superposition of several technological developments related to Cyber-Physical Systems (CPS), Internet of Things (IoT), Internet of Services (IoS) and Data Mining (DM). CPS refers to a new generation of systems with integrated computational and physical capabilities that can interact with humans through many new modalities. The key is the ability to interact with, and expand the capabilities of, the physical world through computation, communication, and control [11]. IoT is defined as the ubiquitous access to entities on the internet for the extension of the physical world through a variety of sensing, detection, identification, location tracking and monitoring equipment [12]. IoS pursuits a similar approach with services instead of physical entities. The integration of these developments promotes the cooperation between the partners alone the entire system. From the perspective of predictive maintenance, Industry 4.0 accelerates and encourages several developments, which will be reviewed and discussed in this section.

\section{A. Cloud computing environment}

Industry 4.0 includes the increasing impact of information and communication technologies on industrial production processes. As one of the driving forces behind Industry 4.0, cloud computing has rapidly emerged as an accepted computing paradigm in many enterprises worldwide due to its flexibility and many other advantages [13]. It can manage shared data from multiple sources efficiently and flexibly in a self-service way and provide a unified service delivery platform for the IoT applications [14]. A cloud-based system provides the technological basis for the provision of data and allows not only creation of community-type services but also building of an open service platform environment which may have features of interactive, collaborative and customizable on demand. From the view point of predictive maintenance, cloud computing environment can efficiently support various smart services and solve several issues such as the memory capacity of equipment, computing power of processor, data security and data fusion from multiple sources.

\section{B. Industrial big data environment}

Under the Industry 4.0 era, another significant development is the combination of intelligent analytics and Cyber-Physical systems for achieving a new type of manufacturing management and factory transformation. The trend behand this combination is the environment of industrial big data. The application of industrial big data has been demonstrated in different areas of manufacturing, including production, supply chain, maintenance and diagnosis, quality management, and energy [15].

In the research fields of maintenance, fault diagnosis and prognosis, appropriate sensor installations and various signals can be applied to monitor the working condition of the equipment. In addition, through comparing the current and historical data with data mining technologies, the degree of potential faults, the reliability of certain components and other useful information may be harvested.

\section{Smart Factory}

Industry 4.0 also facilitate the vision and execution of the idea "Smart Factory", in which cyber-physical systems monitor physical processes, create the virtual copy to represent the physical world and make decentralized decisions. Products may not only provide their identity but also record their properties, history and status via Radio Frequency 
Identification (RFID) technology. Over cloud computing and distributed control, cyber-physical systems communicate and cooperate with each and via the Internet of Services, both internal and cross-organizational services are offered and utilized to make machines self-aware and actively prevents potential performance issues [16].

From the perspective of maintenance, a self-aware and selfmaintained machine system can be considered as a system which can self-assess its own health and degradation, and further use similar information from other peers for smart maintenance decisions to avoid potential faults [1]. To achieve such intelligence, smart analytics may be used at the individual machine or fleet levels. For a mechanical system, self-aware means the capability to assess the current, past or future working condition of a machine, and output the evaluation result. Such health assessment can be performed through data mining technologies to analyse the information collected from the given machine and its ambient environment.

\section{CASE STUDY: REMOTE PREDICTIVE MAINTENANCE SYSTEMS}

In this section, a case study about the application of predictive maintenance will be introduced. This case study comes from a project called Green Monitoring, in which the remote prognostics and monitoring system focused on evaluating and predicting the health of equipment to reduce the maintenance cost on faults, defects and maintenance during the manufacturing process. In addition, RFID technology is applied to identify the components, which may have potential faults. Fig. 2 shows the overall structure of the system, the data is acquired from remote customer site and transported to data mining center for data analysis. Data includes two types: (1) the signals collected from the sensors, which can be used to evaluate the working condition of the equipment or quality of the products. (2) the logistics information in the RFID tags, which can be collected by local RFID readers to identify the components in the equipment.

All the data will be uploaded on the information network, which is controlled by ICB, a software company, for data sharing and publication. The system flowchart is shown in Fig. 3 [17]. After a series of data mining process, including Signal Pre-process and Feature Extraction, the result of fault diagnosis and prognosis can form the Key Performance Indicator (KPI) to evaluate the current working condition and predict the degree of potential faults.

The result of data mining will be recorded and published back to the network for remote condition monitoring and information sharing. The recommended maintenance strategy will be formed in the decision support system according to the prediction or evaluation result. Normally, it requires some relevant optimization and association theories to capture the trade-off between several factors such as maintenance cost, machining accuracy, or defective percentage, and then acquire the optimal decision. In addition, local RFID readers can collect and identify all the RFID tags shown in Fig. 4, which represent the components in the machine.

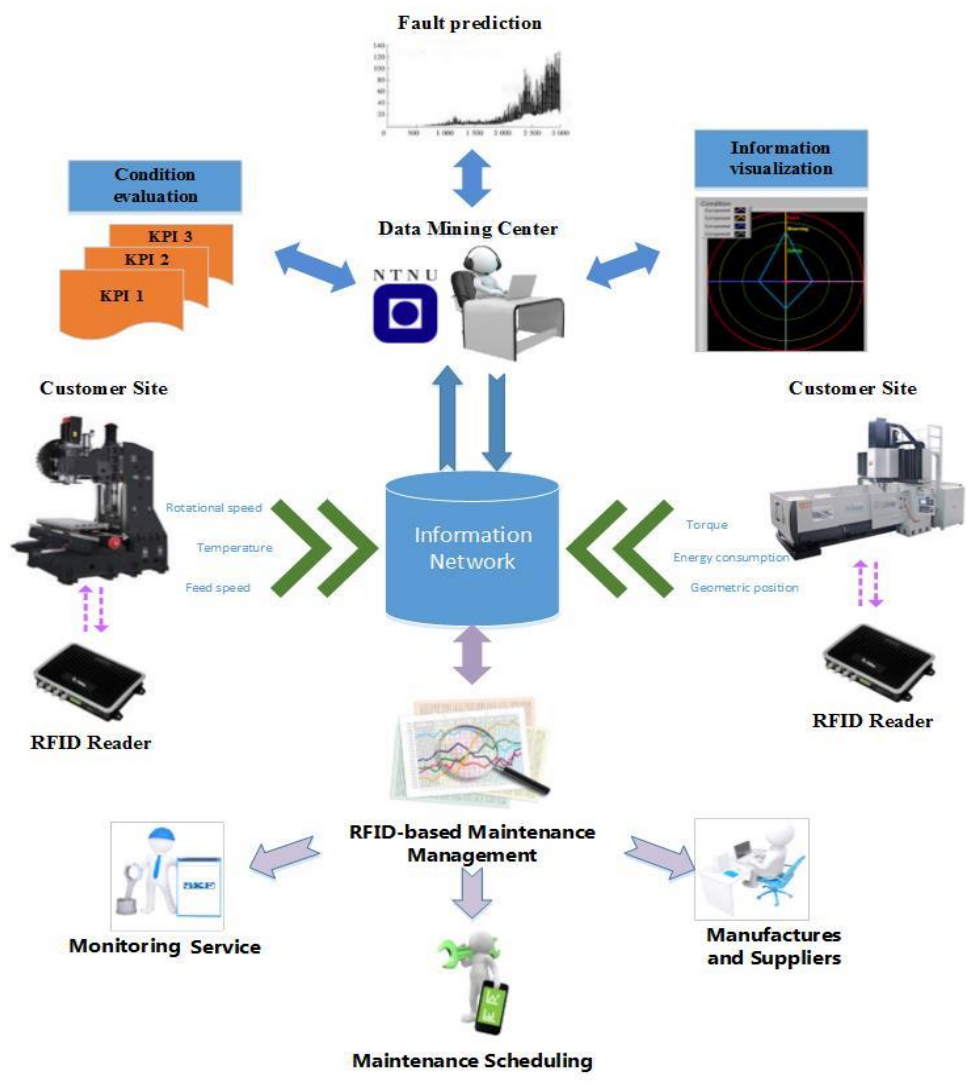

Fig. 2 The Structure of remote predictive maintenance system 


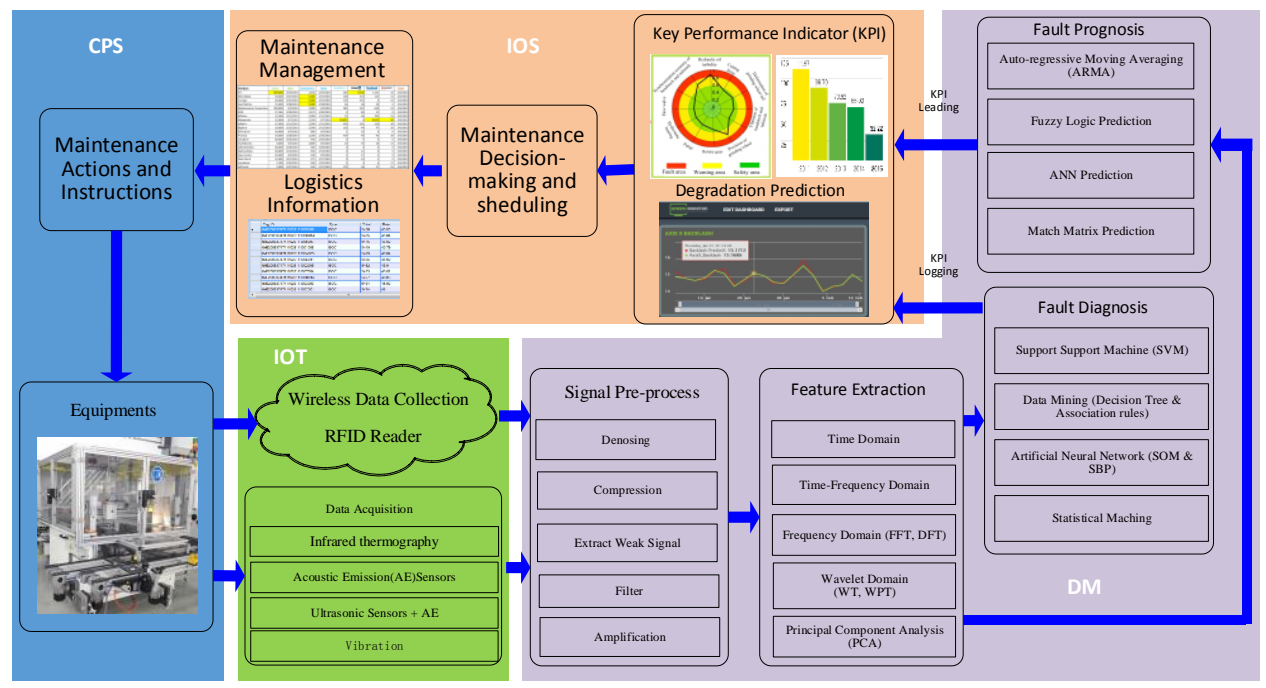

Fig. 3 System flowchart.

Fig. 5 shows a sample of Information in a RFID Tag. Here, Tag ID "0xE200937474111026111005007" can be used for components identity. Normally, the 512 bits user data are reserved for the customized information, which can be converted to 64 ASCII characters. In this case, the condition evaluation related information is written in the user memory of the RFID tag. The information comprises the current working condition, whether has potential faults in the prediction period, when the potential faults may happen, evaluation time and date, in the form of "COND=0 POT=1 FAULT=28.05.16 TIME $=14: 35$ DATE=21.05.16". Since the tag memory is written in the form of hexadecimal, the texts have to be converted to HEX before writing. In this case, the current working condition of the component, which is a spindle in a machine center, is normal $(\mathrm{COND}=0)$. Potential faults are detected (POT=1), which may happen in 28.05.2016 (FAULT $=28.05 .16$ ). In addition, the detection is taken at 14:35 on 21.05.2016 (TIME=14:35 DATE=21.05.16).

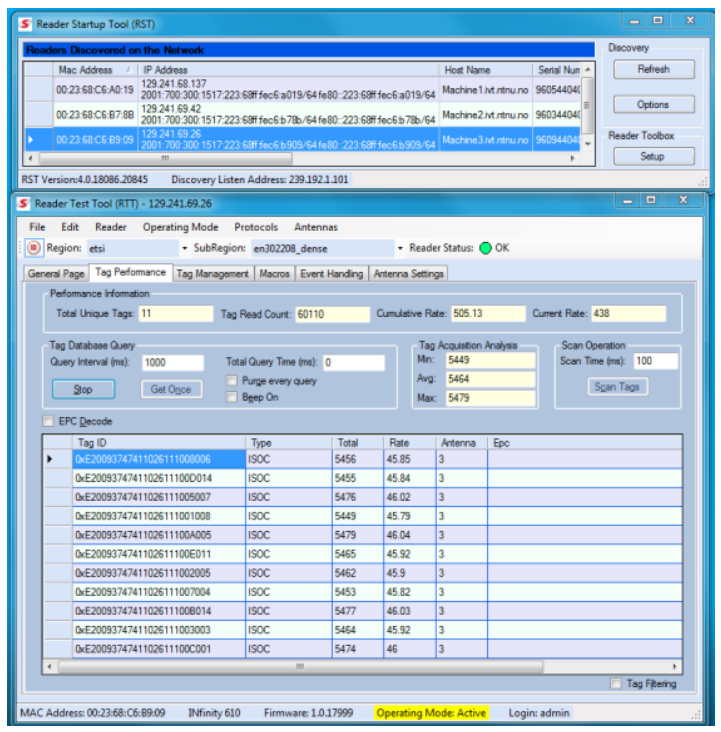

Fig. 4 Record of Tags in RFID Readers

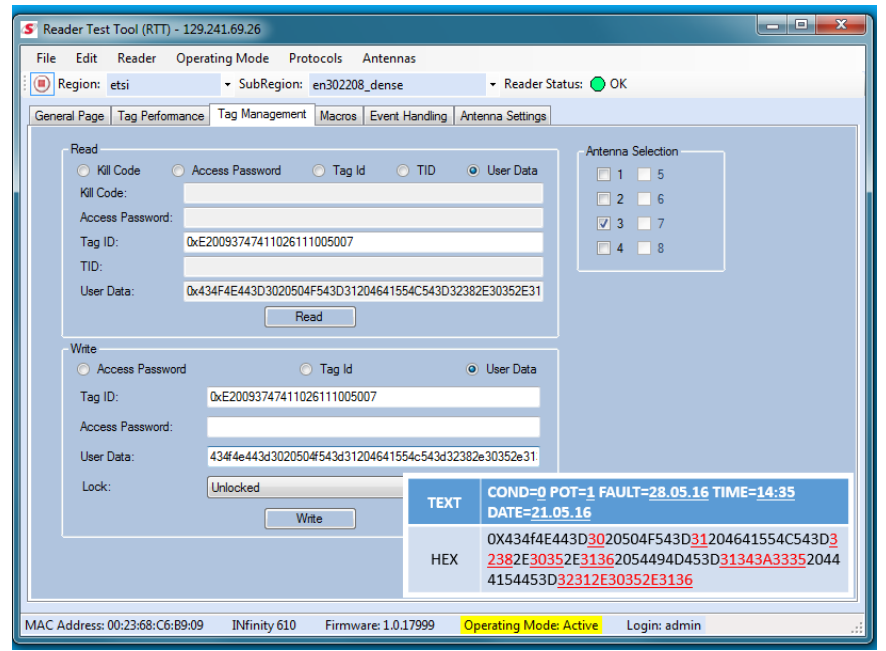

Fig. 5 Information in a RFID Tag.

Combining the result of diagnosis and prognosis with logistics information collected from RFID tags, customers can figure out when, where, which equipment, and which components may have faults.

\section{CONCLUSIONS}

Industry 4.0 promotes the predictive and smart manufacturing in the future industry. In an industry 4,0 factory, the machines are connected as a collaborative community, which generates a great potential for predictive maintenance. This article has investigated the development of predictive maintenance, its technical challenges, and the potential under the environment of Industry 4.0. On one hand, the environment of cloud computing, industrial big data and smart factory is accelerated and encouraged in Industry 4.0 era, which establish the foundation to achieve predictive maintenance. On the other hand, predictive maintenance will play a very important role in future maintenance activities and meet the requirement of 
smart manufacturing, self-aware machine under the terms of Industry 4.0 .

The case study introduced in this paper illustrates how the predictive maintenance and maintenance management can be applied under the environment of cloud computing and industrial big data based on data mining and RFID techniques.

\section{ACKNOWLEDGMENT}

This study has been supported by a grant from Norway through the Norwegian Financial Mechanism 2009-2014, in the frame of the Green Industry Innovation Programme Bulgaria. Moreover, the authors gratefully thank InterConsult Bulgaria (ICB) and Kongsberg Terotech (KTT) for their assistance in the software and data sources used in this study.

\section{REFERENCES}

[1] J. Lee, H.-A. Kao, and S. Yang, "Service innovation and smart analytics for industry 4.0 and big data environment," Procedia CIRP, vol. 16, pp. 3-8, 2014

[2] H. Lasi, P. Fettke, H.-G. Kemper, T. Feld, and M. Hoffmann, "Industry 4.0," Business \& Information Systems Engineering, vol. 6, p. 239, 2014.

[3] A. Hochschild, "EMPIRE OF COTTON A Global History," ed: NEW YORK TIMES 620 8TH AVE, NEW YORK, NY 10018 USA, 2015.

[4] R. Schmidt, M. Möhring, R.-C. Härting, C. Reichstein, P. Neumaier, and P. Jozinović, "Industry 4.0-Potentials for Creating Smart Products: Empirical Research Results," in Business Information Systems, 2015, pp. 16-27.

[5] E. Standard, "Maintenance terminology," in European Committee for Standardization 13306, ed. Brussels, 2001.

[6] K.-S. Wang, Z. Li, J. Braaten, and Q. Yu, "Interpretation and compensation of backlash error data in machine centers for intelligent predictive maintenance using ANNs," Advances in Manufacturing, vol. 3, pp. 97-104, 2015.
[7] M. C. Garcia, M. A. Sanz-Bobi, and J. del Pico, "SIMAP: Intelligent System for Predictive Maintenance: Application to the health condition monitoring of a windturbine gearbox," Computers in Industry, vol. 57, pp. 552-568, 2006.

[8] M. Aljumaili, K. Wandt, R. Karim, and P. Tretten, "eMaintenance ontologies for data quality support," Journal of Quality in Maintenance Engineering, vol. 21, pp. 358-374, 2015.

[9] The Rise of Industrial Big Data. Available: http://leadwise.mediadroit.com/files/19174the_rise_of_industrial_big_d ata_wp_gft834.pdf

[10] J. Liu, D. Djurdjanovic, J. Ni, N. Casoetto, and J. Lee, "Similarity based method for manufacturing process performance prediction and diagnosis," Computers in Industry, vol. 58, pp. 558-566, 8// 2007.

[11] R. Baheti and H. Gill, "Cyber-physical systems," The impact of control technology, vol. 12, pp. 161-166, 2011.

[12] L. W. Chaves and Z. Nochta, "Breakthrough towards the internet of things," in Unique Radio Innovation for the 21st Century, ed: Springer, 2011, pp. 25-38.

[13] J. Bughin, M. Chui, and J. Manyika, "Clouds, big data, and smart assets: Ten tech-enabled business trends to watch," McKinsey Quarterly, vol. 56, pp. 75-86, 2010.

[14] L. Li, L. Xinrui, and L. Xinyu, "Cloud-Based Service Composition Architecture for Internet of Things," Communications in Computer and Information Science, Springer, vol. 312, pp. 559-564, 2012.

[15] P. O'Donovan, K. Leahy, K. Bruton, and D. T. O'Sullivan, "Big data in manufacturing: a systematic mapping study," Journal of Big Data, vol. 2, pp. 1-22, 2015

[16] M. Hermann, T. Pentek, and B. Otto, "Design principles for Industrie 4.0 scenarios: a literature review," Technische Universität Dortmund, Dortmund, 2015.

[17] K. Wang, "Intelligent Predictive maintenance (IPdM) system - Industry 4.0 scenario", editors: K. Wang, Y. Wang, J. O. Strandhagen and T. Yu, Proceedings of Advanced Manufacturing and Automation V, WIT Transaction on Engineering Science, Vol 113, pp. 259-268, 2016 